\title{
Detection of enteroviruses in stools precedes islet autoimmunity by several months: possible evidence for slowly operating mechanisms in virus-induced autoimmunity
}

\author{
Hanna Honkanen $^{1}$ • Sami Oikarinen ${ }^{1,2}$ • Noora Nurminen ${ }^{1}$ • Olli H. Laitinen ${ }^{3,4}$. \\ Heini Huhtala $^{5}$ - Jussi Lehtonen ${ }^{1}$ - Tanja Ruokoranta ${ }^{3}$ - Minna M. Hankaniemi ${ }^{3,4}$. \\ Valérie Lecouturier $^{6}$ • Jeffrey W. Almond ${ }^{6}$ - Sisko Tauriainen ${ }^{7}$ • Olli Simell ${ }^{8,9}$. \\ Jorma Ilonen $^{10}$ • Riitta Veijola ${ }^{11}$ - Hanna Viskari ${ }^{1,12}$ - Mikael Knip ${ }^{13,14,15,16}$. \\ Heikki Hyöty ${ }^{1,2}$
}

Received: 22 August 2016/Accepted: 14 November 2016/Published online: 9 January 2017

(C) Springer-Verlag Berlin Heidelberg 2017

\begin{abstract}
Aims/hypothesis This case-control study was nested in a prospective birth cohort to evaluate whether the presence of enteroviruses in stools was associated with the appearance of islet autoimmunity in the Type 1 Diabetes Prediction and Prevention study in Finland.

Methods Altogether, 1673 longitudinal stool samples from 129 case children who turned positive for multiple islet autoantibodies and 3108 stool samples from 282 matched control children were screened for the presence of enterovirus RNA using RT-PCR. Viral genotype was detected by sequencing.
\end{abstract}

Electronic supplementary material The online version of this article (doi:10.1007/s00125-016-4177-z) contains peer-reviewed but unedited supplementary material, which is available to authorised users.

Hanna Honkanen

hanna.honkanen@uta.fi

1 Department of Virology, University of Tampere, PL100, 33014 Tampereen yliopisto, Finland

2 Fimlab Laboratories, Pirkanmaa Hospital District, Tampere, Finland

3 Vactech Ltd, Tampere, Finland

4 BioMediTech, University of Tampere, Tampere, Finland

5 School of Health Sciences, University of Tampere, Tampere, Finland

6 Sanofi-Pasteur, Marcy L'Etoile, France

7 Department of Virology, University of Turku, Turku, Finland

8 Department of Pediatrics and Research Centre of Applied and Preventive Cardiovascular Medicine, University of Turku, Turku, Finland
Results Case children had more enterovirus infections than control children ( 0.8 vs 0.6 infections per child). Timedependent analysis indicated that this excess of infections occurred more than 1 year before the first detection of islet autoantibodies (6.3 vs 2.1 infections per 10 follow-up years). No such difference was seen in infections occurring less than 1 year before islet autoantibody seroconversion or after seroconversion. The most frequent enterovirus types included coxsackievirus A4 (28\% of genotyped viruses), coxsackievirus A2 (14\%) and coxsackievirus A16 (11\%).

Conclusions/interpretation The results suggest that enterovirus infections diagnosed by detecting viral RNA in stools are

9 Department of Pediatrics and Adolescent Medicine, Turku University Hospital, Turku, Finland

10 Immunogenetics Laboratory, University of Turku and Turku University Hospital, Turku, Finland

11 Department of Pediatrics, PEDEGO Research Unit, Medical Research Center, Oulu University Hospital and University of Oulu, Oulu, Finland

12 Department of Internal Medicine, Tampere University Hospital, Tampere, Finland

13 Children's Hospital, University of Helsinki and Helsinki University Central Hospital, Helsinki, Finland

14 Research Programs Unit, Diabetes and Obesity, University of Helsinki, Helsinki, Finland

15 Department of Pediatrics, Tampere University Hospital, Tampere, Finland

16 Folkhälsan Research Center, Helsinki, Finland 
associated with the development of islet autoimmunity with a time lag of several months.

Keywords Enterovirus · Genotyping · RT-PCR · Stool samples · Type 1 diabetes

$\begin{array}{ll}\text { Abbreviations } \\ \text { DIPP } & \text { Type 1 Diabetes Prediction and Prevention } \\ \text { IAA } & \text { Insulin antibody } \\ \text { ICA } & \text { Islet cell antibody } \\ \text { IA-2A } & \text { Insulinoma-associated protein } 2 \text { antibodies } \\ \text { VP1 } & \text { Viral protein } 1\end{array}$

\section{Introduction}

Type 1 diabetes is caused by an immune-mediated process that damages insulin-producing beta cells in the pancreatic islets. The subclinical phase of the disease can be identified by detecting autoantibodies against islet antigens, including islet cell antibodies (ICAs), insulin antibodies (IAAs), insulinoma-associated protein 2 antibodies (IA-2As), zinc transporter 8 antibodies and GAD antibodies [1].

Enteroviruses have been linked to type 1 diabetes in studies showing an increased frequency of these viruses in the blood and pancreas of diabetic and autoantibody-positive individuals [2-4] and in serological studies showing an increased frequency of enterovirus antibodies in diabetic and autoantibodypositive participants [5-11]. However, this association has not been seen in all studies [8].

Relatively few prospective studies have been carried out even though such studies would be optimal for evaluating the possible role of enterovirus infections in the most important phase of pathogenesis, the initiation of islet autoimmunity. The results from such studies have suggested that enterovirus infections, diagnosed by serology or by direct detection of the virus from blood, are associated with the appearance of islet autoantibodies long before clinical diabetes is diagnosed $[6,9,11,12]$. In contrast, the detection of the virus in stools has not shown such an association $[13,14]$, and in one study in which both blood and rectal swabs were analysed, no association at all was found [15]. However, these negative findings have been based on small cohorts and infrequent sample collection. In addition, the Diabetes and Autoimmunity Study in the Young (DAISY) study in the USA has evaluated the possible role of enterovirus infection in the progression of islet autoimmunity to clinical disease and reported more enterovirus infections detected by the presence of virus in blood but not in stools in children who progressed to diabetes [16].

The current study is the largest study to date in which enteroviruses have been analysed in longitudinal stool samples collected from children who developed signs of a beta cell-damaging process during their prospective observation. Since this study was based on a prospective birth cohort, we were able to analyse time-dependent associations between enterovirus infections and the initiation of the beta celldamaging process.

\section{Methods}

Subjects Serial stool samples were collected longitudinally from children taking part in the Type 1 Diabetes Prediction and Prevention (DIPP) study running in three cities in Finland (Oulu, Tampere and Turku). In this study, children with HLA class II genotypes conferring increased susceptibility to type 1 diabetes (about $12 \%$ of all newborns) are invited to a prospective follow-up starting from birth [17]. All children in the DIPP are regularly screened during the follow-up for the possible presence of diabetes-associated ICAs in serum and, if found to be positive for these, are also tested for biochemically defined autoantibodies including IAA, IA-2A and GAD antibodies. Since the beginning of 2003, all children have been regularly screened for both the biochemically defined autoantibodies and ICAs. ICAs were detected by indirect immunofluorescence, and the three other autoantibodies were quantified with radiolabel-binding assays as previously described [18]. Longitudinal stool samples were collected for viral analyses once a month starting from the age of 3 months and lasting until the age of 2-3 years [19]. The study protocol has been approved by the ethics committees of the University Hospitals in Oulu, Tampere and Turku, and parents gave their written informed consent to their child's participation in the study.

The present study followed a nested case-control design. The case children included 129 children who turned positive for at least two islet autoantibodies and remained autoantibody-positive in all later samples. Seventy-nine case children were positive for one, 30 for two and 9 for three biochemical autoantibodies in the first autoantibody-positive sample. Ninety-seven (75\%) of the children had developed type 1 diabetes by the end of 2015 . The mean age at autoantibody seroconversion was 23 months (range 6-73 months), and diabetes was diagnosed at a mean age of 5 years 10 months (range 10-162 months). The case children were born between 1996 and 2009, and 64\% were boys. From one to five control children were selected for each case child (giving 282 control children altogether), and were matched for area of residence, sex, HLA-DQ genotype and time of birth (+/- 2 months). All control children remained negative for autoantibodies and were non-diabetic at the time when islet autoantibodies appeared in the corresponding case child.

The original HLA screening result was later supplemented by accurate HLA-DR/DQ genotyping [20]. According to 
Ilonen et al [20], different haplotypes have either strong susceptibility, weak susceptibility, neutral susceptibility, weak protection or strong protection properties. Six different genotype risk groups were created based on the properties of individual haplotypes: high risk, moderately increased risk, slightly increased risk, neutral, slightly decreased risk and strongly decreased risk. In the current study, $50 \%$ of case children and $40 \%$ of control children carried the high-risk genotype, $35 \%$ of case and $27 \%$ of control children carried the moderately increased risk genotype, and $15 \%$ of case and $21 \%$ of control children carried the slightly increased risk genotype. The accurate genotyping was not successful in 5\% of control children, and $7 \%$ of control children had a neutral or slightly decreased risk [20].

Detection of enterovirus RNA in stool samples Altogether, 4781 stool samples (1673 samples from 129 case children and 3108 samples from 282 control children) were available for virus analysis (mean 11 samples per child, range 1-34; see electronic supplementary material [ESM] Figs 1 and 2). The mean number of samples did not differ between cases and controls (12 vs 11 samples per child).

All stool samples were screened for the presence of enterovirus RNA using RT-PCR. Analyses were carried out blinded without knowing the case-control status of the child, but the samples from the case child and his or her controls were analysed in the same test run. Both virus-negative and viruspositive control samples were included in every test run.

RT-PCR was carried out using two methods. In both methods, a 10\% (weight/vol) stool suspension was first prepared from the original stool sample in Hanks' solution including gentamicin, penicillin $\mathrm{G}$, amphotericin $\mathrm{B}$ and $4 \%$ BSA. Total nucleic acids were extracted from this suspension for 4099 of the samples using a MagNa Pure extraction robot (Roche, Applied Science, Germany) and Total Nucleid Acid extraction kit (Roche) according to the manufacturer's proto$\mathrm{col}$, and the extracted RNA was then reverse-transcribed, amplified and subjected to detection using a previously described PCR method [21]. The remaining 682 samples were extracted using Qiagen RNeasy 96 kit (Qiagen, Germany) and analysed using real-time RT-PCR using the QuantiTect Probe PCR Kit (Qiagen) as previously described [22]. The real-time PCR was performed according to the instructions on the QuantiTect Probe kit using TaqMan chemistry.

In pilot studies, the two methods were compared using stool samples spiked with different amounts of enterovirusinfected cells and were found to have comparable sensitivity (data not shown). In addition, the same method was always used to analyse stool samples from a particular case child and from his or her controls, thus excluding biases in case-control comparison in a scenario where the methods could have had minor differences in their sensitivity.
Sequencing All samples that were positive for enterovirus RNA in RT-PCR analyses were genotyped by sequencing the viral protein 1 (VP1) region of the viral genome [23]. The obtained sequences were blasted against the National Center for Biotechnology Information non-redundant nucleotide database. If the child had the same genotype in consecutive samples, these samples were interpreted as one infection by the given genotype.

Statistical analyses The frequency of enterovirus infections during the follow-up was adjusted to the duration of the follow-up time by assuming that each stool sample covered enterovirus infections during the preceding month (a 1 month period of viral shedding into the stools). Thus, each stool sample represented a 1 month follow-up time. The number of infections per 10 follow-up years was used to present the data.

In time-dependent analyses, the time of sample draws for each child was adjusted to the time of birth or the time when islet autoantibodies first appeared. Additional analyses were carried out for a cohort that was harmonised for the time of sample collection by including only those samples which were taken during the same calendar month from the case child and his or her matched controls, and by including only such casecontrol pairs whose complete HLA-DR/DQ genotypes were defined. In addition, the number of infections per child was adjusted for the number of samples available from the child.

Conditional logistic regression analysis was used in the comparisons of the matched case-control groups using Stata 13.1 (StataCorp, College Station, TX, USA) and R 3.2.2 (www.r-project.org) to compute ORs with their 95\% CIs. A $p$ value of $<0.05$ was considered statistically significant. The OR in the current study describes how much one positive follow-up month increased the risk of a child turning autoantibody-positive during the follow-up.

Ethics The study was approved by the ethics committees of the participating university hospitals, and the parents gave their written informed consent to the participation in the study.

\section{Results}

Enterovirus RNA was detected in 370 (7.7\%) of all the 4781 stool samples. A clear seasonal pattern was seen as the samples collected during March to July showed the lowest frequency of enterovirus positivity (Fig. 1). The rate of enterovirus infection was similar in children younger than 6 months of age and in those aged 6-18 months or 18-24 months (5.2, 5.2 and 5.9 infections per 10 follow-up years, respectively) but declined to 2.0 infections per 10 follow-up years in older children (Fig. 2). The proportion of enterovirus-positive samples did not differ between boys and girls $(8.9 \%$ vs $7.0 \%)$ or 
Fig. 1 Percentage of enteroviruspositive samples according to the month of sample collection. Black bars, case children; white bars, control children. EV, enterovirus

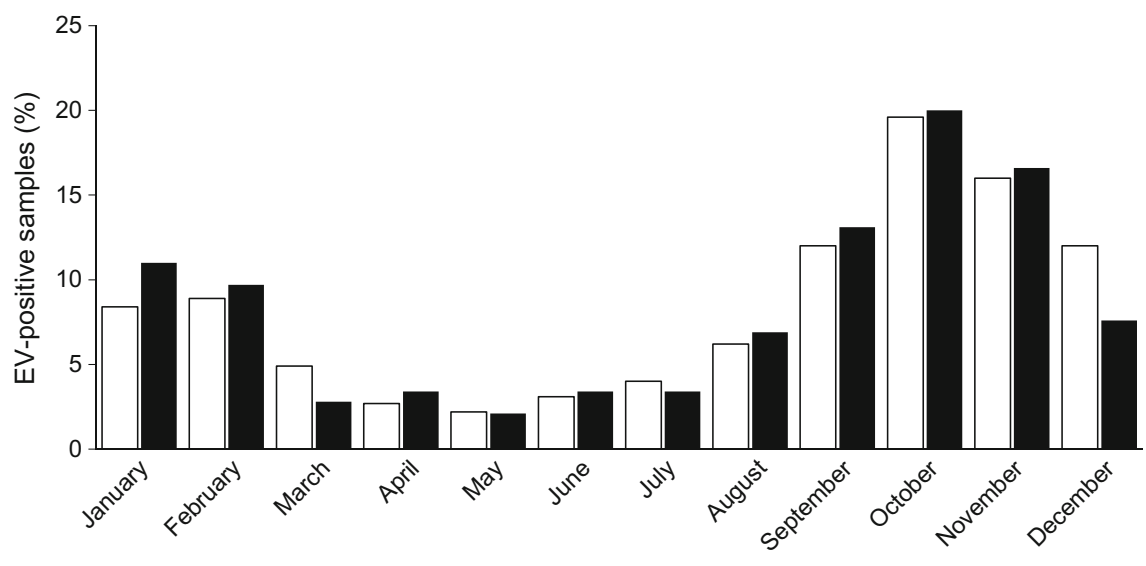

between different HLA-DR/DQ groups (high-risk genotype group $7.4 \%$, moderately increased risk genotype group $8.7 \%$, and slightly increased genotype group $7.4 \%$ ).

Altogether, 108 infections were diagnosed in the 129 case children and 169 infections in the 282 control children during the whole follow-up (mean 0.8 vs. 0.6 infections per child). This difference was also seen in infections that occurred prior to the appearance of autoantibodies ( $0.6 \mathrm{vs} 0.4$ infections per child). This difference was most obvious in the youngest and oldest age groups (Fig. 2 and ESM Fig. 2). These results were also similar when only the 109 case children who developed islet autoantibodies early, before the age of 3 years, and their 250 controls were included in these analyses ( 0.6 vs 0.5 infections per child). The first enterovirus-positive samples were detected, on average, at the age of 11 months among both cases (range 3-26 months) and controls (range 3-25 months).

Time-dependent analyses showed that the excess of infections in case children occurred more than 12 months before the first autoantibody-positive sample was taken. During this time period, an average of 0.62 infections were diagnosed per case child compared with 0.33 infections per control child, corresponding to 6.3 vs 2.1 infections per 10 follow-up years,

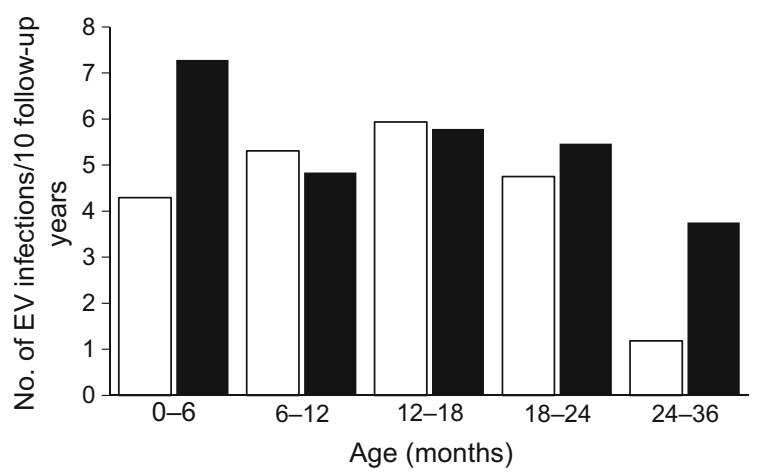

Fig. 2 Infections per 10 follow-up years in different age groups in case and control children. Number of samples in each age group: 0-6 months, 848 ; 6-12 months, $1579 ; 12-18$ months, $1211 ; 18-24$ months, 862 ; 24 36 months, 279. The number of enterovirus infections in these age groups did not differ statistically. Black bars, case children; white bars, control children. EV, enterovirus respectively (233 children; OR $1.03,95 \%$ CI $1.00,1.06$, $p=0.023)$. Altogether, $33.8 \%$ (26/77) of the case children and $21.2 \%(33 / 156)$ of the control children had at least one infection during that period $(\mathrm{OR}=2.3,95 \%$ CI $1.1,5.0$, $p=0.033$ ). The same difference was seen among case children who turned autoantibody-positive before the age of 3 years: 0.5 vs 0.2 infections per child, corresponding to 5.7 vs 1.5 infections per 10 follow-up years (182 children; OR 1.03, $95 \%$ CI 1.00, 1.07, $p=0.035$; Fig. 3). At least one infection was diagnosed in $30.4 \%$ (17/56) of these cases compared with $15.1 \%(19 / 126)$ of their controls (OR 3.0, 95\% CI 1.2, 7.6, $p=0.022$ ). The excess of infections was also seen when only those samples that were collected during exactly the same calendar month from both the case child and his or her matched control child/children were included in these analyses (233 children; OR 1.03, 95\% CI 1.00, 1.06, $p=0.042$ ), as well as when the number of infections per child was adjusted for the number of samples available from the child (OR 2.0, 95\% CI 1.0, 4.0, $p=0.043$ ). In addition, the difference was seen when only those case-control pairs were included whose completed HLA-DR/DQ genotypes matched (176 children; OR $1.06,95 \%$ CI 1.01, 1.10, $p=0.01$ ) and when only those cases who developed type 1 diabetes and their controls were included (172 children; OR 1.04, CI 1.00, 1.08, $p=0.029$ ). In contrast to these early infections, the infections that occurred later, within a year prior to autoantibody seroconversion or

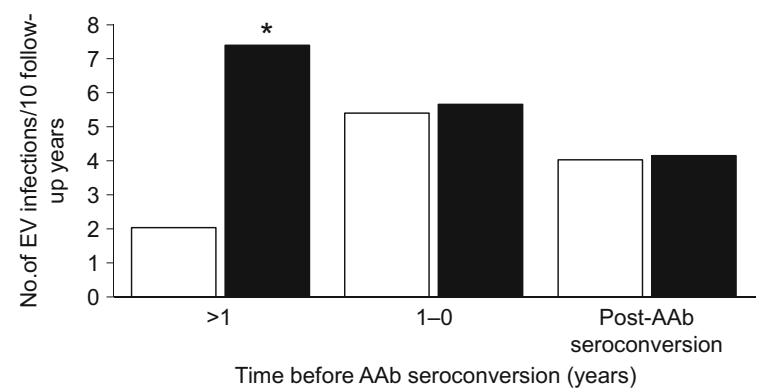

Fig. 3 Number of enterovirus infections per 10 follow-up years in children who turned islet autoantibody-positive before the age of 3 years and their controls. Black bars, case children; white bars, control children. $* p=0.035$. AAb, autoantibody; $\mathrm{EV}$, enterovirus 
after autoantibody seroconversion, were not associated with islet autoimmunity (Fig. 3). The results were similar in both sexes (data not shown). The proportion of strongly enterovirus-positive samples did not differ between case and control children (data not shown).

The sequence analysis of the VP1 region of the viral genome was successful for 244 of all 370 enterovirus-positive samples (90 case samples and 154 control samples, success rate $66 \% ; 73 \%$ in strong positives and $27 \%$ in weak positives). Sequence analysis identified 277 individual infections and provided the exact viral genotype in 174 (63\%) of them (63 infections in case children and 111 infections in control children). Twenty-five per cent of the cases and $27 \%$ of the controls had two consecutive samples positive for the same virus strain, and $8 \%$ of the cases and $5 \%$ of the controls had three or more consecutive samples positive for the same virus strain, reflecting the long excretion period of the virus $(p=0.078$ for three or more samples).

Altogether, 25 different enterovirus types were detected and species A enteroviruses were the predominating types (Table 1). None of the individual enterovirus types showed statistically significant association with islet autoimmunity. This was true when all detected infections were analysed as well as when only infections preceding the first detection of autoantibodies were analysed. Some enterovirus types were only observed in case children (coxsackieviruses A14 and B2, echovirus 13) and some only in control children (coxsackievirus B4, echoviruses 4, 7, 9, 25 and 30, and enteroviruses 68 and 90), but these occurred rarely (Table 1).

\section{Discussion}

This is the largest study carried out so far in which the occurrence of enteroviruses has been studied in longitudinal stool samples collected from children who developed signs of a beta cell-damaging process, and has been compared with results from carefully matched control children. Overall, in the current study, enterovirus infections were detected more frequently in case than in control children. The excess of infections preceded the appearance of autoantibodies, paralleling the findings from our previous prospective studies in the DIPP study, the Trial to Reduce IDDM in the Genetically at Risk (TRIGR) pilot and the Childhood Diabetes in Finland (DiMe) cohorts, in which enterovirus infections were documented by antibody assays and detection of viral RNA from serum [6, 9, 11, 24-26]. In addition, the Norwegian Environmental Triggers of Type 1 Diabetes (MIDIA) study also observed a peak in enterovirus RNA in blood at the time of autoantibody seroconversion [12]. Altogether, these findings suggest that enterovirus infections might play a role in the initiation of the beta cell-damaging process.
Table 1 Number of different enterovirus genotypes in case and control children and their relative proportion (\%) from all successfully genotyped enterovirus infections

\begin{tabular}{|c|c|c|c|c|}
\hline \multirow[t]{2}{*}{ Genotype } & \multicolumn{2}{|c|}{ All case and control children } & \multicolumn{2}{|c|}{$\begin{array}{l}\text { Children who progressed to } \\
\text { clinical type } 1 \text { diabetes and } \\
\text { their controls }\end{array}$} \\
\hline & $\begin{array}{l}\text { Case } \\
n=129(\%)\end{array}$ & $\begin{array}{l}\text { Control } \\
n=282(\%)\end{array}$ & $\begin{array}{l}\text { Case } \\
n=97(\%)\end{array}$ & $\begin{array}{l}\text { Control } \\
n=221(\%)\end{array}$ \\
\hline CVA2 & $7(11.1)$ & $14(12.6)$ & $6(12.0)$ & $10(11.6)$ \\
\hline CVA3 & $2(3.2)$ & $1(0.9)$ & $1(2.0)$ & $1(1.2)$ \\
\hline CVA4 & $16(25.4)$ & $25(22.5)$ & $13(26.0)$ & $20(23.3)$ \\
\hline CVA5 & $4(6.3)$ & $4(3.6)$ & $2(4.0)$ & $4(4.7)$ \\
\hline CVA6 & $4(6.3)$ & $6(5.4)$ & $4(8.0)$ & $1(1.2)$ \\
\hline CVA9 & $2(3.2)$ & $4(3.6)$ & $2(4.0)$ & $2(2.3)$ \\
\hline CVA10 & $3(4.8)$ & $6(5.4)$ & $2(4.0)$ & $5(5.8)$ \\
\hline CVA14 & $2(3.2)$ & $0(0)$ & $2(4.0)$ & $0(0)$ \\
\hline CVA16 & $7(11.1)$ & $15(13.5)$ & $5(10.0)$ & $12(14.0)$ \\
\hline $\mathrm{CVA}^{\mathrm{a}}$ & $1(1.6)$ & $0(0)$ & $1(2.0)$ & $0(0)$ \\
\hline CVB2 & $1(1.6)$ & $0(0)$ & $0(0)$ & $0(0)$ \\
\hline CVB3 & $2(3.2)$ & $5(4.5)$ & $2(4.0)$ & $4(4.7)$ \\
\hline CVB4 & $0(0)$ & $4(3.6)$ & $0(0)$ & $1(1.2)$ \\
\hline CVB5 & $4(6.3)$ & $4(3.6)$ & $2(4.0)$ & $4(4.7)$ \\
\hline ECHO4 & $0(0)$ & $1(0.9)$ & $0(0)$ & $1(1.2)$ \\
\hline ECHO7 & $0(0)$ & $1(0.9)$ & $0(0)$ & $1(1.2)$ \\
\hline ECHO9 & $0(0)$ & $3(2.7)$ & $0(0)$ & $2(2.3)$ \\
\hline ECHO11 & $2(3.2)$ & $1(0.9)$ & $2(4.0)$ & $1(1.2)$ \\
\hline ECHO13 & $1(1.6)$ & $0(0)$ & $1(2.0)$ & $0(0)$ \\
\hline ECHO18 & $2(3.2)$ & $1(0.9)$ & $2(4.0)$ & $1(1.2)$ \\
\hline ECHO25 & $0(0)$ & $2(1.8)$ & $0(0)$ & $2(2.3)$ \\
\hline ECHO30 & $0(0)$ & $3(2.7)$ & $0(0)$ & $3(3.5)$ \\
\hline EV68 & $0(0)$ & $4(3.6)$ & $0(0)$ & $4(4.7)$ \\
\hline EV71 & $3(4.8)$ & $6(5.4)$ & $3(6.0)$ & $6(7.0)$ \\
\hline EV90 & $0(0)$ & $1(0.9)$ & $0(0)$ & $1(1.2)$ \\
\hline All & 63 & 111 & 50 & 86 \\
\hline
\end{tabular}

$\mathrm{CV}$, coxsackievirus; ECHO, echovirus; $\mathrm{EV}$, enterovirus

${ }^{\mathrm{a}}$ The exact genotype was not identified

Only two previously published studies have evaluated the association between enteroviruses and type 1 diabetes in prospective birth cohorts by detecting enteroviruses in longitudinal stool sample series $[13,14]$. Neither of those studies found any difference in enterovirus infections between children who developed islet autoimmunity or type 1 diabetes and matched control children. One of these studies included 22 case and 82 control children [13], and the other included 27 case and 53 control children [14]. One additional cohort study detected enteroviruses from both blood samples and rectal swabs, showing no difference between 26 case and 39 control children [15], but later reported that enterovirus infections might be associated with the progression of islet autoimmunity to diabetes [16]. Altogether, these studies were based on a 
considerably smaller number of children than in our study, which included 129 case and 282 control children. In addition, one of these studies analysed stool samples only until the age of 1 year [13] and another study collected samples with long time intervals at the age of 9, 15 and 24 months, and annually thereafter [15]. These aspects limited their power to detect a time-dependent association between enterovirus infections and islet autoimmunity.

The circulation of enteroviruses differs between populations and in different time periods, which may influence their association with type 1 diabetes in different studies. For example, we have proposed that the relatively low incidence of enterovirus infections in Finland may make Finnish children more susceptible to the possible diabetogenic effect of enteroviruses [27]. The lower frequency of 'background' enterovirus infections may also make it easier to detect the association between enterovirus infection and type 1 diabetes in Finland. The slightly lower frequency of enterovirus-positive samples in the current study (7.7\%) compared with the German and Norwegian cohort studies (9-13\%) $[13,14]$ is also in line with a lower frequency of enterovirus infections in Finland. The observed seasonal pattern of enterovirus positivity fits with the known enterovirus seasonality observed in countries with a temperate climate.

The excess of enterovirus infections occurred more than 12 months before islet autoantibody seroconversion. As the average time interval from last autoantibody-negative serum sample to the first autoantibody-positive serum sample was 6 months (range 2-20 months), it can be concluded that the actual time lag is about 9 months or more. Taking into account the fact that the mean age at the first autoantibody-positive sample was 23 months, it is evident that most of these infections have occurred at a very young age, when children are more susceptible to enterovirus infections. It is known that enteroviruses can spread to the pancreatic islets in such young infants $[3,28]$. However, since stool samples were collected only until the age of 3 years, further studies would be needed to determine whether the presence of enteroviruses in stools is associated with islet autoimmunity in older age groups.

The time lag from excessive infections to islet autoantibody seroconversion was longer than in our previous study, which was based on the detection of enterovirus RNA in serum [9]. The reason for this difference is not known, but it may be due to the fact that these two cohorts of children were different, sharing only eight case children. It is also possible that the detection of viral RNA in serum reflects later stages of the infection, for example viral replication in internal organs such as pancreas. On the other hand, it is not known how closely the time of autoantibody seroconversion reflects the time when the beta cell-damaging process actually starts, and how long a lag there is from the time of the triggering insults to the appearance of autoantibodies. These aspects may also vary considerably between individuals. Mouse models have suggested that GAD65 autoantibodies appear within 8 weeks after coxsackievirus B4 infection [29], while in humans the time lag can be longer, particularly if viral persistency and/or interactions with other risk factors contribute to the process. In any case, the present study argues against a rapid lytic effect of enteroviruses on beta cells, but rather suggests a possible involvement of viral persistence and/or immune-mediated mechanisms in enterovirus-induced diabetes in humans.

The enterovirus genotype was identified by sequence analysis in $66 \%$ of all enterovirus-positive samples. Species A enteroviruses predominated, and $70 \%$ of all successfully sequenced samples contained a coxsackie A virus. The most frequent individual coxsackievirus genotypes were A4 (in 28\% of samples), A2 (14\%) and A16 (11\%). Only a minority of the samples contained a coxsackie B group virus $(11 \%)$ or an echovirus $(10 \%)$. The high prevalence of species A enteroviruses is in line with the results from another birth cohort study [30]. In addition, our previous serological study in the DIPP cohort indicated a high prevalence of neutralising antibodies against species $\mathrm{A}$ enteroviruses [11], and the results of the current study are in line with these observations. On the other hand, the genotype distribution observed in the current study differs from that seen in France and the Netherlands, where a majority of the enterovirus infections have been found to be caused by species $B$ enteroviruses [31, 32]. However, these studies were carried out among hospitalised patients and also included respiratory samples [31, 32].

When the occurrence of different enterovirus genotypes was compared between case and control groups, none of them showed an association with beta cell autoimmunity. However, even if this study is the largest study of this kind, it clearly lacks statistical power to analyse this question for most of the enterovirus types detected. This is emphasised by the fact that a relatively large proportion of detected enteroviruses remained untyped due to the low titre of the virus (42\% in case children and $34 \%$ in control children). For example, coxsackie B and certain other species B viruses that have been linked to type 1 diabetes $[2,5,11,19,33-40]$ were detected in only a few samples. This may reflect the fact that coxsackie B infections are mainly respiratory in nature and hence not detected in stools as frequently as in respiratory samples. For example, type B1, which is associated with an increased risk of islet autoimmunity in serological studies [10, 11], has most commonly been detected in respiratory samples in surveillance studies [41]. The duration of virus excretion into stools may also differ between different enterovirus types, and studies based on relatively long sample intervals can miss a large proportion of infections with short virus excretion periods. In any case, further studies analysing a large cohort of children using both serological and direct enterovirus detection methods and blood, stool and respiratory samples are needed to get a complete picture of the occurrence of different enterovirus types in case and control children. This kind of systematic screening of enterovirus types 
would be crucially important for the development of vaccines against these viruses.

One limitation of the present study is that it was carried out in one country, and further studies are needed to find out whether a similar association can be seen in other populations. Another limitation is the lack of respiratory samples. Such samples have not been collected in the DIPP study, but many enterovirus types, such as EV68 and coxsackie B viruses, often cause respiratory infections, and their detection in stools may lead to a marked underestimation of their true frequency $[42,43]$. The case and control children were tightly matched for the time of birth, age at sampling, sex and area of residence, which reduced the risk of the possible effect of confounding factors. In addition, they were matched for HLAconferred risk of type 1 diabetes because HLA genes can influence anti-enterovirus immunity [44]. However, we cannot exclude the possibility that some other non-HLA genes could influence the detection of enteroviruses, even though we consider this unlikely since previous studies have not found any major effect of such genes on enterovirus positivity in stools [45-47].

In conclusion, the present study suggests that enterovirus infections in young children are associated with the appearance of islet autoantibodies with a time lag of about 1 year. This finding supports previous observations from other prospective studies suggesting that enterovirus infections may play a role in the initiation of the beta cell-damaging process.

Acknowledgements The authors wish to thank T. Kuusela, M. Kekäläinen, E. Jalonen, P. Keränen, M. Ovaskainen and M. Lumme from the virology department of the University of Tampere, Finland, for their excellent technical assistance.

Data availability The data that support the findings of this study are available on reasonable request from the guarantor HHy. The data are not publicly available as they contain information that could compromise research participants' consent.

Funding This study was funded by the Scientific Foundation of the City of Tampere, Finnish Cultural Foundation, Pirkanmaa Regional fund, Finnish Funding Agency for Technology and Innovation, Competitive Research Funding of the Tampere University Hospital, JDRF, Diabetes Research Foundation, Academy of Finland, Diabetes Research Foundation in Finland, Sigrid Juselius Foundation, Reino Lahtikari Foundation and European Commission (Persistent Virus Infection in Diabetes Network [PEVNET] Frame Programme 7, Contract No. 261441). In addition, it was partly funded by Sanofi Pasteur and Vactech Ltd. The study sponsor was involved in the design of the study and provided editorial assistance when submitting the report for publication.

Duality of interest $\mathrm{HHy}$ and $\mathrm{MK}$ are minor $(5 \%)$ shareholders and members of the board of Vactech Ltd, which develops vaccines against picornaviruses. Companies owned by the families of HHy and MK are also shareholders of Vactech Ltd. No other potential conflicts of interest relevant to this article were reported.
Contribution statement HHo designed the study, researched the data and wrote the manuscript. HV, $\mathrm{NN}$ and $\mathrm{SO}$ designed the study, researched the data and reviewed/edited manuscript. HHu and JL performed the statistical analysis and reviewed manuscript. OHL, TR, MMH, VL, JWA, ST, OS, JI, RV and MK designed the study and reviewed/edited the manuscript. HHy designed the study and contributed to the discussion, and reviewed/edited the manuscript. HHy is the guarantor of this work and, as such, had full access to all the data in the study and takes responsibility for the integrity of the data and the accuracy of the data analysis. All authors have approved the final version of the article.

\section{References}

1. Lampasona V, Liberati D (2016) Islet autoantibodies. Curr Diab Rep 16:53

2. Yoon JW, Austin M, Onodera T, Notkins AL (1979) Isolation of a virus from the pancreas of a child with diabetic ketoacidosis. N Engl J Med 300:1173-1179

3. Richardson SJ, Willcox A, Bone AJ, Foulis AK, Morgan NG (2009) The prevalence of enteroviral capsid protein vp1 immunostaining in pancreatic islets in human type 1 diabetes. Diabetologia 52:1143-1151

4. Yeung WC, Rawlinson WD, Craig ME (2011) Enterovirus infection and type 1 diabetes mellitus: systematic review and metaanalysis of observational molecular studies. BMJ 342:d35

5. Gamble DR, Kinsley ML, FitzGerald MG, Bolton R, Taylor KW (1969) Viral antibodies in diabetes mellitus. Br Med J 3:627-630

6. Hyoty H, Hiltunen M, Knip M et al (1995) A prospective study of the role of coxsackie B and other enterovirus infections in the pathogenesis of IDDM. Childhood Diabetes in Finland (DiMe) Study Group. Diabetes 44:652-657

7. Helfand RF, Gary HE Jr, Freeman CY, Anderson LJ, Pallansch MA (1995) Serologic evidence of an association between enteroviruses and the onset of type 1 diabetes mellitus. Pittsburgh Diabetes Research Group. J Infect Dis 172:1206-1211

8. Green J, Casabonne D, Newton R (2004) Coxsackie B virus serology and type 1 diabetes mellitus: a systematic review of published case-control studies. Diabet Med 21:507-514

9. Oikarinen S, Martiskainen M, Tauriainen S et al (2011) Enterovirus RNA in blood is linked to the development of type 1 diabetes. Diabetes 60:276-279

10. Oikarinen S, Tauriainen S, Hober D et al (2014) Virus antibody survey in different European populations indicates risk association between coxsackievirus B1 and type 1 diabetes. Diabetes 63:655-662

11. Laitinen $\mathrm{OH}$, Honkanen $\mathrm{H}$, Pakkanen O et al (2014) Coxsackievirus $\mathrm{B} 1$ is associated with induction of beta-cell autoimmunity that portends type 1 diabetes. Diabetes 63:446-455

12. Cinek O, Stene LC, Kramna L et al (2014) Enterovirus RNA in longitudinal blood samples and risk of islet autoimmunity in children with a high genetic risk of type 1 diabetes: the MIDIA study. Diabetologia 57:2193-2200

13. Simonen-Tikka ML, Pflueger M, Klemola P et al (2011) Human enterovirus infections in children at increased risk for type 1 diabetes: the Babydiet study. Diabetologia 54:2995-3002

14. Tapia G, Cinek O, Rasmussen T et al (2011) Human enterovirus RNA in monthly fecal samples and islet autoimmunity in Norwegian children with high genetic risk for type 1 diabetes: the MIDIA study. Diabetes Care 34:151-155

15. Graves PM, Rotbart HA, Nix WA et al (2003) Prospective study of enteroviral infections and development of beta-cell autoimmunity. 
Diabetes Autoimmunity Study in the Young (DAISY). Diabetes Res Clin Pract 59:51-61

16. Stene LC, Oikarinen S, Hyoty H et al (2010) Enterovirus infection and progression from islet autoimmunity to type 1 diabetes: the Diabetes and Autoimmunity Study in the Young (DAISY). Diabetes 59:3174-3180

17. Nanto-Salonen K, Kupila A, Simell S et al (2008) Nasal insulin to prevent type 1 diabetes in children with HLA genotypes and autoantibodies conferring increased risk of disease: a double-blind, randomised controlled trial. Lancet 372:1746-1755

18. Kukko M, Kimpimaki T, Korhonen S et al (2005) Dynamics of diabetes-associated autoantibodies in young children with human leukocyte antigen-conferred risk of type 1 diabetes recruited from the general population. J Clin Endocrinol Metab 90:2712-2717

19. Salminen KK, Vuorinen T, Oikarinen S et al (2004) Isolation of enterovirus strains from children with preclinical type 1 diabetes. Diabet Med 21:156-164

20. Ilonen J, Kiviniemi M, Lempainen J et al (2016) Genetic susceptibility to type 1 diabetes in childhood - estimation of HLA class II associated disease risk and class II effect in various phases of islet autoimmunity. Pediatr Diabetes 17(Suppl 22):8-16

21. Lonnrot M, Sjoroos M, Salminen K, Maaronen M, Hyypia T, Hyoty H (1999) Diagnosis of enterovirus and rhinovirus infections by RT-PCR and time-resolved fluorometry with lanthanide chelate labeled probes. J Med Virol 59:378-384

22. Honkanen H, Oikarinen S, Pakkanen O et al (2013) Human enterovirus 71 strains in the background population and in hospital patients in Finland. J Clin Virol 56:348-353

23. Nix WA, Oberste MS, Pallansch MA (2006) Sensitive, seminested PCR amplification of VP1 sequences for direct identification of all enterovirus serotypes from original clinical specimens. J Clin Microbiol 44:2698-2704

24. Salminen K, Sadeharju K, Lonnrot M et al (2003) Enterovirus infections are associated with the induction of beta-cell autoimmunity in a prospective birth cohort study. J Med Virol 69:91-98

25. Sadeharju K, Hamalainen AM, Knip M et al (2003) Enterovirus infections as a risk factor for type I diabetes: virus analyses in a dietary intervention trial. Clin Exp Immunol 132:271-277

26. Lempainen J, Tauriainen S, Vaarala O et al (2012) Interaction of enterovirus infection and cow's milk-based formula nutrition in type 1 diabetes-associated autoimmunity. Diabetes Metab Res Rev 28:177-185

27. Viskari H, Ludvigsson J, Uibo R et al (2004) Relationship between the incidence of type 1 diabetes and enterovirus infections in different European populations: results from the EPIVIR project. J Med Virol 72:610-617

28. Jenson AB, Rosenberg HS, Notkins AL (1980) Pancreatic islet-cell damage in children with fatal viral infections. Lancet 2:354-358

29. Chatterjee NK, Hou J, Dockstader P, Charbonneau T (1992) Coxsackievirus B4 infection alters thymic, splenic, and peripheral lymphocyte repertoire preceding onset of hyperglycemia in mice. J Med Virol 38:124-131

30. Simonen-Tikka ML, Hiekka AK, Klemola P et al (2012) Early human enterovirus infections in healthy Swedish children participating in the PRODIA pilot study. J Med Virol 84:923-930

31. Tan CY, Ninove L, Gaudart J et al (2011) A retrospective overview of enterovirus infection diagnosis and molecular epidemiology in the public hospitals of Marseille, France (1985-2005). PLoS One 6: e18022
32. van der Sanden SM, Koopmans MP, van der Avoort HG (2013) Detection of human enteroviruses and parechoviruses as part of the national enterovirus surveillance in the Netherlands, 1996-2011. Eur J Clin Microbiol Infect Dis 32:1525-1531

33. Champsaur H, Dussaix E, Samolyk D, Fabre M, Bach C, Assan R (1980) Diabetes and Coxsackie virus B5 infection. Lancet 1:251

34. Wagenknecht LE, Roseman JM, Herman WH (1991) Increased incidence of insulin-dependent diabetes mellitus following an epidemic of Coxsackievirus B5. Am J Epidemiol 133:1024-1031

35. Clements GB, Galbraith DN, Taylor KW (1995) Coxsackie B virus infection and onset of childhood diabetes. Lancet 346:221-223

36. Andreoletti L, Hober D, Hober-Vandenberghe C et al (1997) Detection of coxsackie B virus RNA sequences in whole blood samples from adult patients at the onset of type I diabetes mellitus. J Med Virol 52:121-127

37. Roivainen M, Knip M, Hyoty H et al (1998) Several different enterovirus serotypes can be associated with prediabetic autoimmune episodes and onset of overt IDDM. Childhood Diabetes in Finland (DiMe) Study Group. J Med Virol 56:74-78

38. Chehadeh W, Kerr-Conte J, Pattou F et al (2000) Persistent infection of human pancreatic islets by coxsackievirus B is associated with alpha interferon synthesis in beta cells. J Virol 74:10153-10164

39. Yin H, Berg AK, Westman J, Hellerstrom C, Frisk G (2002) Complete nucleotide sequence of a Coxsackievirus B-4 strain capable of establishing persistent infection in human pancreatic islet cells: effects on insulin release, proinsulin synthesis, and cell morphology. J Med Virol 68:544-557

40. Dotta F, Censini S, van Halteren AG et al (2007) Coxsackie B4 virus infection of beta cells and natural killer cell insulitis in recent-onset type 1 diabetic patients. Proc Natl Acad Sci U S A 104:5115-5120

41. Khetsuriani N, Lamonte-Fowlkes A, Oberst S, Pallansch MA, Centers for Disease Control and Prevention (2006) Enterovirus surveillance-United States, 1970-2005. MMWR Surveill Summ 55:1-20

42. Fujimoto T, Shinohara M, Ito M et al (2007) Detection of dualinfected cases of adenoviruses and coxsackieviruses type B by real-time PCR but not by the conventional viral culture technique. Clin Lab 53:605-609

43. Lu QB, Wo Y, Wang HY et al (2014) Detection of enterovirus 68 as one of the commonest types of enterovirus found in patients with acute respiratory tract infection in China. J Med Microbiol 63:408-414

44. Sadeharju K, Knip M, Hiltunen M, Akerblom HK, Hyoty H (2003) The HLA-DR phenotype modulates the humoral immune response to enterovirus antigens. Diabetologia 46:1100-1105

45. Witso E, Tapia G, Cinek O, Pociot FM, Stene LC, Ronningen KS (2011) Polymorphisms in the innate immune IFIH1 gene, frequency of enterovirus in monthly fecal samples during infancy, and islet autoimmunity. PLoS One 6:e27781

46. Witso E, Cinek O, Tapia G, Rasmussen T, Stene LC, Ronningen KS (2012) HLA-DRB1-DQA1-DQB1 genotype and frequency of enterovirus in longitudinal monthly fecal samples from healthy infants. Viral Immunol 25:187-192

47. Witso E, Cinek O, Tapia G et al (2015) Genetic determinants of enterovirus infections: polymorphisms in type 1 diabetes and innate immune genes in the MIDIA study. Viral Immunol 28:556-563 